\title{
Multikinase inhibitors to treat hepatocellular carcinoma failures to sorafenib-time has come for a better approach
}

\author{
Saleh A. Alqahtani ${ }^{1,2}$, Massimo Colombo ${ }^{3}$ \\ ${ }^{1}$ Liver Transplant Unit, King Faisal Specialist Hospital \& Research Centre, Riyadh, Saudi Arabia; ${ }^{2}$ Division of Gastroenterology and Hepatology, \\ Johns Hopkins University, Baltimore, MD, USA; ${ }^{3}$ Center of Translational Research in Hepatology, Humanitas Hospital, Rozzano, Italy \\ Correspondence to: Massimo Colombo. Center of Translational Research in Hepatology, Humanitas Hospital, Rozzano, Italy. \\ Email: mcolombo46@yahoo.it. \\ Comment on: Kudo M, Kang YK, Park JW, et al. Regional Differences in Efficacy, Safety, and Biomarkers for Second-Line Axitinib in Patients with \\ Advanced Hepatocellular Carcinoma: From a Randomized Phase II Study. Liver Cancer 2018;7:148-64.
}

Submitted Apr 09, 2020. Accepted for publication Apr 21, 2020.

doi: $10.21037 /$ atm-2020-79

View this article at: http://dx.doi.org/10.21037/atm-2020-79

Hepatocellular carcinoma (HCC), a highly vascularized tumor of the liver, is the third leading cause of death worldwide (1). The incidence of HCC has been soaring in most countries, especially in developing countries in Asian and sub-Saharan Africa than in developed countries in Europe and North America. In Asia (excluding Japan) and Africa, hepatitis B is the primary cause of HCC. Standard treatment options are established for early diagnosed HCC that include surgical resection, locoregional therapy, ablation, and liver transplantation. However, a majority of patients are recognized with advanced HCC, which has been a challenge to treat with many limitations, and the need to establish an effective treatment regimen seems critical $(2,3)$.

Systemic treatment of advanced HCC has evolved substantially over the past decade following the advent of two anti-vascular endothelial growth factors (VEGF) regimens, sorafenib in 2008 and lenvatinib in 2018, which proved to confer survival benefits to patients with advanced disease, including patients with metastases $(4,5)$. A few months ago, the first-line treatment of patients with advanced HCC had been revolutionized following the successful outcome of a phase III randomized controlled trial (RCT) in Asia where the combination of two recombinant monoclonal antibodies, the programmed cell death-1 (PD-1) ligand antagonist atezolizumab and the anti-VEGF-A bevacizumab outperformed the standard of care sorafenib in terms of overall survival (OS), with an odds ratio of 0.58 at a $95 \%$ confidence interval of $0.43-0.79$ coupled with a clinically meaningful delay in deterioration of patient- reported quality of life (6). While this immune-oncology (IO) regimen stands as a practice-changing treatment of advanced HCC which offers the advantage of bypassing the bottleneck of the high rates of toxicity and contraindications of anti-VEGF regimens, it still requires to be validated in western patients and special subgroups like patients with organ transplant, autoimmune diseases, immunosuppression, and decompensated liver. On top of this, the advent of the first-line therapy of HCC based on IOs poses pragmatic problems concerning strategies of retreatment, since nowadays retreatment embraces rescue regimens that have been validated in patients with a sorafenib failure, only $(2,3)$. The pillars of the current algorithm for retreatment, in fact, are the multikinase inhibitor regorafenib for sorafenib tolerant patients, with a projected 26 months of median survival with the sequence sorafenib-regorafenib, another multikinase inhibitor cabozantinib for sorafenib intolerant patients, and the anti-angiogenic monoclonal antibody ramucirumab for patients with more than $400 \mathrm{ng}$ serum level of alfafetoprotein (7-9). In the US, two monoclonal PD-1check point inhibitors pembrolizumab and nivolumab, and a combination of nivolumab and the CTLA-4 immune checkpoint inhibitor ipilimumab have also been approved as second-line therapy of HCC (10). Of note, all these developments came through following an intense rollover of clinical research and in the face of a large number of randomized trials where experimental regimens failed to achieve the primary endpoint of conferring survival 
benefits compared to placebo (11). While the high rate of failures of RCTs assessing systemic treatments of HCC likely depends in part on a limited therapeutic index of most multikinase inhibitors and the frequent association of HCC with hepatic impairment, treatment efficacy of most experimental drugs was obscured by the enrolment of clinically heterogeneous patients with different patterns of tumor progression during sorafenib therapy, a variable entailing substantial differences in survival. As a matter of facts, the survival of patients under sorafenib who harbor a tumor growing outside the liver or invading the portal vessels is substantially shorter than that of patients in whom a preexisting nodule in the liver grows without spreading into the portal system (12). With this as a background, it is little surprise that an anti-VEGF drug like axitinib, tested in a phase II RCT enrolling a clinically heterogeneous population of non-responders to sorafenib with respect to the pattern of HCC progression, did fail to provide survival benefits compared to placebo (13). As in most malignancies, angiogenesis, via several pathways, including VEGF/ VEGF receptor (VEGFR) signaling, is thought to play a critical role in the progression of advanced HCC (14). Antiangiogenic medications are effective in treating most metastatic cancers $(15,16)$, but efficacy is limited with several caveats, including side effects and intolerance. Studies on several inhibitors of angiogenic signals, including the multikinase inhibitor brivanib (17) and the monoclonal antibody ramucirumab (18), or the mTOR inhibitor everolimus (19) as a second-line treatment in patients with sorafenib-refractory and -intolerance in HCC, failed to show favorable results.

Axitinib, a potent, selective inhibitor of VEGF receptors $1-3$, has been the standard for the treatment of secondline terminal malignant renal cell carcinoma (20). Although robust clinical evidence for the use of axitinib for secondline treatment in advanced HCC was lacking, its invitro efficacy and perceived clinical success has made axitinib attractive for clinical trials in metastatic HCC. In phase II clinical trials, axitinib was found to have promising efficacy in patients who previously received VEGF but demands further investigation (21).

A previous study by Kang et al. evaluated the efficacy and safety of axitinib in a randomized phase II clinical trial in comparison with placebo plus best supportive care (BSC) in second-line treatment of advanced HCC in patients with localized or metastatic HCC (22). The subjects including patients with HCC and Child-Pugh Class A, who failed to respond or were intolerant to prior antiangiogenic therapy, were stratified by tumor invasion and geographic region (Asian/non-Asian). The results did not show an improved OS over placebo/BSC in the overall population or the stratified subgroups. However, axitinib/BSC resulted in longer progression-free survival (PFS), time to tumor progression, and clinical benefit rate with manageable toxicity. An exploratory subgroup analysis showed improved OS when patients intolerant to previous antiangiogenic treatment were excluded-more preferentially in Asian patients than non-Asians.

The current study by Kudo et al. (13) includes 78 patients enrolled from non-Asian countries and 124 patients from Asian countries [Japan $(n=37)$, Korea $(n=36)$, and mainland China/Hong Kong/Taiwan (CHT) (n=51)] who were randomized to axitinib/BSC ( $\mathrm{n}=134$ patients, 51 non-Asians and 83 Asians) versus placebo/BSC $(68,27$ non-Asians and 41 Asians). The aim was to investigate the differences in OS and PFS among Asian and non-Asians populations and to explore patient populations based on demographics and baseline characteristics. Additionally, the authors explored potential biomarkers with prognostic values of baseline microRNAs and serum soluble proteins, providing therefore a welcome contribution to the literature on axitinib for advanced HCC and a step forward in the quality of study design.

Unfortunately, the report by Kudo et al. (13) confirms with prior observations by Kang et al. (22) that axitinib conferred no significant improvement in OS over placebo arm in both Asian and non-Asian populations. After exclusion of patients intolerant to first-line antiangiogenic therapy, an exploratory analysis showed favorable OS in axitinib/BSC arm over placebo/BSC, especially in patients from Asia than from non-Asia. A key observation was a longer OS in axitinib/BSC treatment arm in patients from Japan than from Korea and CHT, whereas patients from Japan had an overall better tolerance to axitinib/BSC than patients from Korea, CHT, and non-Asia. A range of disparities in the adverse effects (AE) was seen among both Asian and non-Asian patient groups-more in axitinib/ BSC arm than placebo/BSC arm. AEs, such as asthenia, were more commonly seen in patients from non-Asia, whereas Asian patients frequently exhibited diminished appetite, hand-foot syndrome, and proteinuria. Also, severe AEs were more frequently seen in patients from non-Asia than Asia, indicating a higher tolerance to axitinib in Asian populations. Interestingly, the percentage of patients who had AEs leading to axitinib dose reductions was higher in Asia than non-Asia ( $41 \%$ vs. 24\%), whereas more patients discontinued the treatment due to AEs in non-Asia than 
Asia groups. Among Asian groups, more patients from Korea required frequent dose reductions than in Japan or CHT groups ( $60 \%$ vs. $42 \%$ or $26 \%$ ), yet fewer Korean patients had AEs leading to axitinib discontinuation (8\% vs. $23 \%$ or $32 \%$ ). Nevertheless, the percentage of patients who had overall higher axitinib dose reductions was in Japan compared with patients from Korea, CHT, and non-Asia (69\% vs. $44 \%$ vs. $35 \%$ vs. $37 \%$ ), whereas Japanese patients had the lowest dose of axitinib than patients from other groups. No patient from Japan had an increase in axitinib dose during the treatment regime.

In general, there were no prominent disparities among patient populations of this study. However, patients from Asia and Japan had a higher incidence of hepatitis B and hepatitis $\mathrm{C}$ infections respectively, and a higher percentage of patients from Asia, especially Japan, had minor differences in baseline extrahepatic spreads with intrahepatic tumors and tumor vascular invasions between the axitinib/ BSC and placebo/BSC treatment arms. Differences in baseline characteristics of HCC were also seen among patients from Asia and non-Asia, confirming geographical differences in HCC risk factors.

While these differences make the data challenging to interpret, the majority of the patients in both the treatment arms received systematic therapy with sorafenib, and a higher percentage of patients in the placebo/BSC arm were intolerant to sorafenib. Worthy to be noted is the high prevalence of patients from Asia in both the treatment arms who had more than one prior locoregional therapy compared to non-Asian patients. There were also significant geographical differences in the follow-up therapy, with a more proportion of patients from Japan undergoing poststudy locoregional therapy and subsequently receiving follow up systematic therapy, mostly with sorafenib, than patients from other groups.

The PFS was longer in the axitinib/BSC arm than in placebo/BSC arm in patients from Asia than non-Asia, an observation that aligns with previous study results, with patients from Japan predominantly having longer PFS than patients from Korea and CHT. While it is unclear if the differences in the follow-up therapies and aggressive axitinib treatment modifications contributed to the favorable OS and PFS in patients from Japan, it is worthy to note that a previous randomized phase III trial of ramucirumab in advanced HCC patients showed similar disparities in treatment practices and baseline characteristicscharacteristics. In a subgroup analysis, a subgroup analysis, favoring patients from Japan (23) over the total population (18). Another study that evaluated the regional differences in sorafenib-treated patients with HCC had similar findings-reflecting a real-world setting for clinical trials (24).

Intriguingly, Kudo et al. (13) identified four circulating miRNAs associated with longer OS in both Asian and nonAsian populations. Three miRNAs (hsa-miR-5684, hsamiR-1224-5p, and hsa-miR-513c-5p) were associated with longer OS in patients from Asia than non-Asia, and the other miRNA (hsa-miR-6075) was detected in patients with longer OS from non-Asia than Asia. The data is preliminary, and further evaluation confirming this data could help develop efficient diagnostic tools in the prognosis and treatment of advanced HCC in the future. The previous study by Kang et al. (22) also showed an association between a low baseline serum level of E-selectin or SDF-1 and longer OS among axitinib/BSC treated patients over placebo/BSC in HCC. Besides, the study also reported a lower-than-median baseline level of IL-6, E-selectin, IL-8, Ang-2, MIF, or soluble c-MET to be a potential prognostic factor associated with OS (21). Consistent with this data, the authors of the current subgroup analysis found an important association between lowerthan- or equal-to-median baseline serum levels of SDF-1 and longer OS in Asian patients treated with axitinib/BSC over placebo/BSC. However, no association was observed in non-Asian patients, suggesting regional disparities in the prognosis of HCC.

The median OS in the axitinib/BSC treatment arm favored Asians, uniquely Japanese than non-Asians in the exploratory analysis, but interestingly, the median OS was shorter in placebo/BSC arm in patients from Korea (4.6 months) and Japan (6.3 months) than from CHT (9.4 months) or non-Asia (11.2 months). Given patients from Japan in both the treatment arms had the highest poststudy locoregional and/or systemic therapy, it is intriguing to know why they had less favorable OS than CHT and non-Asia in the placebo/BSC treatment arm. Incidentally, patients from the placebo/BSC arm had a reduced treatment duration than axitinib/BSC in all the groups.

Remarkably, both the studies by Kang et al. (22) and the current analysis by Kudo et al. (13) observed a favorable OS and PFS in axitinib/BSC treatment arm after exclusion of patients who did not tolerate prior antiangiogenicsuggesting axitinib to be a potential second-line treatment option for patients who progress well with the first-line systemic therapy.

This study has some limitations, the most critical one being that it was an exploratory analysis, not to speak of 
differences in baseline characteristics, frequency of followup therapy, lack of analysis of hepatic function, and small sample size for some Asian subgroups (only 11 patients each were enrolled in the placebo/BSC arm for Japan and Korea) which could have impacted the study results. Although this study identified potential biomarkers that include four circulating miRNAs, which could help predict better treatment outcomes in advanced HCC patients, differences in tumor characteristics between subgroups and a small number of patients analyzed for biomarkers makes it challenging. Validation of this data and further investigation in a large group of patients are required, despite differences in demographics, baseline characteristics, aggressive post-study treatment with sorafenib and/or locoregional therapy, and axitinib dose modifications, which could have potentially favored a better OS in axitinib/BSC over placebo/BSC in Japanese patients. It is unclear if differences can safely be attributed to genomic variations, clinical heterogeneity of HCC or differences in VEGF/VEGFR signaling pathway responses between Asians and nonAsians, all warranting further investigation. In the future, it may be worth exploring whether appropriate axitinib dose modifications could favor extension of treatment duration and have a favorable impact on clinical outcomes.

\section{Acknowledgments}

Funding: None.

\section{Footnote}

Provenance and Peer Review: This article was commissioned by the editorial office, Annals of Translational Medicine. The article did not undergo external peer review.

Conflicts of Interest: Both authors have completed the ICMJE uniform disclosure form (available at http://dx.doi. org/10.21037/atm-2020-79). The authors have no conflicts of interest to declare.

Ethical Statement: The authors are accountable for all aspects of the work in ensuring that questions related to the accuracy or integrity of any part of the work are appropriately investigated and resolved.

Open Access Statement: This is an Open Access article distributed in accordance with the Creative Commons Attribution-NonCommercial-NoDerivs 4.0 International
License (CC BY-NC-ND 4.0), which permits the noncommercial replication and distribution of the article with the strict proviso that no changes or edits are made and the original work is properly cited (including links to both the formal publication through the relevant DOI and the license). See: https://creativecommons.org/licenses/by-nc-nd/4.0/.

\section{References}

1. Bray F, Ferlay J, Soerjomataram I, et al. Global cancer statistics 2018: GLOBOCAN estimates of incidence and mortality worldwide for 36 cancers in 185 countries. CA Cancer J Clin 2018;68:394-424.

2. EASL Clinical Practice Guidelines: Management of hepatocellular carcinoma. J Hepatol 2018;69:182-236.

3. Heimbach JK, Kulik LM, Finn RS, et al. AASLD guidelines for the treatment of hepatocellular carcinoma. Hepatology 2018;67:358-80.

4. Llovet JM, Ricci S, Mazzaferro V, et al. Sorafenib in advanced hepatocellular carcinoma. N Engl J Med 2008;359:378-90.

5. Kudo M, Finn RS, Qin S, et al. Lenvatinib versus sorafenib in first- line treatment of patients with unresectable hepatocellular carcinoma: a randomised phase 3 noninferiority trial. Lancet 2018;391:1163-73.

6. Cheng AL, Qin S, Ikeda M, et al. IMbrave150: Efficacy and safety results from a ph III study evaluating atezolizumab (atezo) + bevacizumab (bev) vs sorafenib (Sor) as first treatment (tx) for patients (pts) with unresectable hepatocellular carcinoma (HCC) LBA 3 ESMO Singapore 2019. Ann Oncol 2019;30:ix186-7.

7. Bruix J, Qin S, Merle P, et al. Regorafenib for patients with hepatocellular carcinoma who progressed on sorafenib treatment (RESORCE): a randomised, double-blind, placebo-controlled, phase 3 trial. Lancet 2017;389:56-66.

8. Abou-Alfa GK, Meyer T, Cheng AL, et al. Cabozantinib in patients with advanced and progressing hepatocellular carcinoma. N Engl J Med 2018;379:54-63.

9. Zhu AX, Kang YK, Yen CJ, et al. Ramucirumab after sorafenib in patients with advanced hepatocellular carcinoma and increased $\alpha$ - fetoprotein concentrations (REACH-2): a randomised, double- blind, placebocontrolled, phase 3 trial. Lancet Oncol 2019:20:282-96.

10. Yau T, Kang YK, Kim TY, et al. Nivolumab + Ipilimumab Combination Therapy in Patients With Advanced Hepatocellular Carcinoma: Results From Check Mate 040. Presentation at American Society of Clinical Oncology congress, May 31-June 4. J Clin Oncol 2019;37:abstr 4012. 
11. Bruix J, da Fonseca LG, Reig M. Insights into the success and failure of systemic therapy for hepatocellular carcinoma. Nat Rev Gastroenterol Hepatol 2019;16:617-30.

12. Reig M, Rimola J, Torres F, et al. Post progression survival of patients with advanced hepatocellular carcinoma: rationale for second-line trial design. Hepatology 2013;58:2023-31.

13. Kudo M, Kang YK, Park JW, et al. Regional Differences in Efficacy, Safety, and Biomarkers for Second-Line Axitinib in Patients with Advanced Hepatocellular Carcinoma: From a Randomized Phase II Study. Liver Cancer 2018;7:148-64.

14. Zhu AX, Duda DG, Sahani DV, et al. HCC and angiogenesis: possible targets and future directions. Nat Rev Clin Oncol 2011;8:292-301.

15. European Association for the Study of the Liver. European Association for the Study of the Liver. EASL Clinical Practice Guidelines: Management of hepatocellular carcinoma. J Hepatol 2018;69:182-236

16. Terashima T, Yamashita T, Takata N, et al. Postprogression survival and progression-free survival in patients with advanced hepatocellular carcinoma treated by sorafenib. Hepatol Res 2016;46:650-6.

17. Llovet JM, Decaens T, Raoul JL, et al. Brivanib in patients with advanced hepatocellular carcinoma who were intolerant to sorafenib or for whom sorafenib failed: results from the randomized phase III BRISK-PS study. J Clin Oncol 2013;31:3509-16.

Cite this article as: Alqahtani SA, Colombo M. Multikinase inhibitors to treat hepatocellular carcinoma failures to sorafenib-time has come for a better approach. Ann Transl Med 2020;8(19):1255. doi: 10.21037/atm-2020-79
18. Zhu AX, ParkJO, Ryoo BY, et al. Ramucirumab versus placebo as second-line treatment in patients with advanced hepatocellular carcinoma following first-line therapy with sorafenib (REACH): a randomised, double-blind, multicentre, phase 3 trial. Lancet Oncol 2015;16:859-70.

19. Zhu AX, Kudo M, Assenat E, et al. Effect of everolimus on survival in advanced hepatocellular carcinoma after failure of sorafenib: the EVOLVE-1 randomized clinical trial. JAMA 2014;312:57-67.

20. INLYTA $^{\circledR}$ (axitinib) prescribing information. Pfizer. c2012. Available online: http://labeling.pfizer.com/ShowLabeling. aspx? $\mathrm{id}=759$

21. McNamara MG, Le LW, Horgan AM, et al. A phase II trial of second-line axitinib following prior antiangiogenic therapy in advanced hepatocellular carcinoma. Cancer 2015;121:1620-7.

22. Kang YK, Yau T, Park JW, et al. Randomized phase II study of axitinib versus placebo plus best supportive care in second-line treatment of advanced hepatocellular carcinoma. Ann Oncol 2015;26:2457-63.

23. Kudo M, Hatano E, Ohkawa S, et al. Ramucirumab as second-line treatment in patients with advanced hepatocellular carcinoma: Japanese subgroup analysis of the REACH trial. J Gastroenterol 2017;52:494-503.

24. Kudo M, Lencioni R, Marrero JA, et al. Regional differences in sorafenib-treated patients with hepatocellular carcinoma: GIDEON observational study. Liver Int 2016;36:1196-205. 